\title{
Determinants of Anemia in Pregnant Women with Emphasis on Intestinal Helminthic Infection at Sher- Ethiopia Hospital, Ziway, Southern Ethiopia
}

\author{
Girum Tefera \\ Department of Medical Biotechnology, Tehran University of Medical Sciences, Iran \\ *Corresponding Author: tgirumt@gmail.com
}

Copyright (C) 2014 Horizon Research Publishing All rights reserved.

\begin{abstract}
Back ground: Anemia is a common and serious problem in pregnancy. Parasitic infections contribute to iron deficiency anemia in pregnant women. The objective of the study was to assess determinants of anemia among pregnant women with emphasis on intestinal helminthic infection at Sher- Ethiopia hospital, Ziway, southern Ethiopia. Method: A cross-sectional study was carried out among pregnant women who came for their antenatal care follow up at Sher-Ethiopia hospital, Ziway, Southern Ethiopia from April, 1/2014 - June 30/ 2014. A total of 748 pregnant women were screened for intestinal parasitic infection using direct microscopy and formol ether concentration techniques and level of hemoglobin was determined using hematocrit screening technique. Other determinants of anemia during pregnancy were also assessed by using structured questionnaire. Data were analyzed using SPSS for windows version 16.0. Statistical tests were performed at the level of significance of $5 \%$. Result: Prevalence of intestinal parasite infection in pregnant women was $58.2 \%$. Out of 436 intestinal parasite infected women $176(40.4 \%)$ had more than one intestinal parasitic infection. Prevalence of anemia in this study subjects was $51.9 \%$ and the mean hematocrit level was $34 \%$. Anemic women were 10 times likely to have hookworm plus other intestinal helminthic infection ( $\mathrm{p}$ value $=.000), 2$ times likely to have birth interval less than two years ( $\mathrm{p}$ value $=.018), 2$ times likely not to have shoe wearing habit $(\mathrm{p}$ value $=.045)$ and 2 times likely not having taken iron during pregnancy ( $\mathrm{p}$ value $=.025)$. Conclusion: From the result it can de concluded that all antenatal care attendants should be screened for anemia and intestinal helminthic infection at their first visit and those anemic and helminthic infected women should be treated properly.
\end{abstract}

Keywords Pregnant Women; Anemia, Antenatal Care, Parasite, Hematocrit, Sher-Ethiopia Hospital

\section{Introduction}

Anemia is one of the most widespread public health problems, especially in developing countries. It impaired cognitive development, reduced physical work capacity and in severe cases increased risk of mortality particularly during prenatal period (1). Anemia in pregnant women is defined as hemoglobin levels less than $11 \mathrm{~g} / \mathrm{dl}$ (2). It is usually caused by iron deficiency, which is the most common nutrient deficiency in the world. It has been estimated that, at any one time in developing countries, half of the population (mainly children and women of reproductive age) is affected by anemia (3). During pregnancy, approximately $75 \%$ all anemia diagnosed are due to iron deficiency (4).

An estimated 44 million pregnant women are infected with hookworm worldwide, with 7.5 million in sub-Saharan African alone. Hookworm infection is considered a major health threat to adolescent girls and women of reproductive age, with adverse effects on the outcome of pregnancy (5). Hookworm infections induce deficiencies of iron, total energy, protein and possible folate and zinc (6)

Severe iron deficiency anemia during pregnancy has been linked to increased maternal mortality, impaired lactation prematurity and low birth weight (5). Estimates in Kenya and Nepal suggest that hookworm infection causes 30 percent and 41 percent, respectively, of moderate or severe cases of anemia among pregnant women (hemoglobin level, $<9 \mathrm{~g} / \mathrm{dl}$. The association between hookworm infection and anemia is greatest in multigravidas (5).Studies in Africa and Asia reported a higher prevalence of anemia and its association with women of age $<20$ years, third trimester of pregnancy, rural residents and multi- parous women (7). Anemia in pregnancy is also related to different socio-demographic, dietary and economic factors $(20,29)$. Mother's age $<20$ years, educational status, economic position, and antenatal care were significantly associated with anemia during pregnancy in a study conducted in India (8).

In Ethiopia, anemia is the most frequent morbidity among pregnant women with the prevalence ranging from 23$66.5 \%(9,25)$. There is an urban rural difference in the prevalence of anemia. As indicated by studies in Asendabo 
and Mettue, anemia among pregnant women was consistently higher in the rural women compared to the urban counterparts $(9,25)$.

\section{Materials and Method}

\subsection{Study Design}

A cross-sectional study was conducted to assess determinants of anemia with emphasis on helminthic infection in pregnant women attending antenatal care at Sher-Ethiopia hospital.

\subsection{Study Area}

The study was conducted from April 1, 2014 to June 30, 2014 at Sher-Ethiopia hospital, Ziway. Ziway is a town and separate woreda in central Ethiopia. It is located on the road connecting Addis Ababa to Nairobi in the East of the Oromia

Region of Ethiopia, Ziway has a latitude and longitude of $7^{\circ} 56^{\prime} \mathrm{N} 38^{\circ} 43^{\prime} \mathrm{E}$ with an elevation of 1643 meters above sea level. Adjacent to Lake Ziway, the economy of the town is based on fishing and horticulture. Ziway is also home to a prison and a caustic soda factory.

The 2007 national census reported a total population for this woreda of 120,862 , of whom 60,379 were men and 60,483 were women; 4,506 or $3.73 \%$ of its population were urban dwellers. The majority of the inhabitants said they were Muslim, with $91.04 \%$ of the population reporting they observed this belief, while $7.82 \%$ of the population practiced Christianity, the rest are Protestants.

\subsection{The Study Population}

Pregnant women attending antenatal follow up at Sher-Ethiopia hospital were a study population for this particular study.

\subsection{Study Subjects}

Pregnant women attending Sher-Ethiopia hospital for their antenatal care during the study period who met the inclusion criteria were the study subjects.

\subsection{Sample Size Determination and Sampling Techniques}

\subsubsection{Sample Size}

The required sample size for this study was calculated based on the prevalence rate of $67 \%$ of hookworm infection reported from the previous study done on intestinal helminthic infection and schistomiasis in the community in Delo Awraja Bale administrative Region Southern Ethiopia (28), and the $95 \%$ confidence interval and 5\% marginal error, sample size (n) was determined using the following statistical formula (Danile, 1995).

$$
\mathrm{n}=\frac{Z \mathrm{P}^{2}(1-\mathrm{P})}{\mathrm{D}^{2}} \mathrm{D}=\text { margin of error between the sample }
$$

and the population.

$\mathrm{n}=$ sample size

$\mathrm{Z}=95 \%$ confident interval

$\mathrm{P}=$ prevalence rate of $134 \%$ based on the previous study

$\mathrm{n}=\frac{1.96^{2} \times 1.34(1-1.34):}{0.05^{2}}=680$

By adding $10 \%$ for none response the final sample size was 748.

\subsubsection{Sampling Techniques}

Women who came for antenatal care follow up were assessed and those pregnant women who met the inclusion criteria during the study period included in this study.

\subsection{Inclusion Criteria}

- $\quad$ Resident in the study area

- Pregnant women

- Not having received anthelminthic treatment for the last 6 months

- $\quad$ Given consent to participate

\subsection{Exclusion Criteria}

- $\quad$ Pregnant women who are severely sick because of medical conditions (diabetes, renal or cardiorespiratory diseases, and chronic hypertension) for which follow up are required. Such type of pregnant women was screened by a medical doctors at Sher-Ethiopia hospital.

- Non pregnant women

\subsection{Data Collection Procedure}

\subsubsection{Questionnaires}

A structured questionnaire was used to obtain socio-demographic information, present and past history in pregnant women, environmental related factors and dietary habit. The questionnaire was developed in English and then translated into Amharic language (official language of Ethiopia). Public health nurses who can speak the local language (oromigna) obtained training on data collection procedure for this particular study to attain standardization and maximize interviewer reliability. The data collectors were regularly supervised by the principal investigator for proper data collection.

\subsubsection{Parasitological Examination}

\subsubsection{Collection of Stool Specimen}

Stool specimen containers were given to each pregnant woman with toilet tissue paper and clean applicator stick after questionnaire administration to bring fresh stool specimen.

Orientation was given to the women on how to collect sufficient amount and contamination free stool specimen. Women were requested to bring the stool sample 
immediately to process and examine within two hours. The laboratory technician checked whether sufficient amount of stool specimen was collected or not during receiving of sample.

\subsubsection{Stool Specimen Examination Methods}

\subsubsection{Direct Microscopy Method}

Stool smear was prepared using saline for direct microscopic identification of intestinal helminthes and protozoa infection. Two slides were prepared for each pregnant woman. Direct smear was examined by $10 \mathrm{X}$ and 40 $\mathrm{X}$ microscopic magnifications.

\subsubsection{Concentration Method}

Formol ether sedimentation technique was used following standard operational procedure to concentrate wide range parasites from stool specimen with minimum damage to their morphology (26). Smear was prepared from fecal deposit and examined by $10 \mathrm{X}$ and $40 \mathrm{X}$ microscopic magnifications.

\subsubsection{Blood Specimen Examination}

\subsubsection{Blood Film Examination}

Pregnant women were screened for the presence of malaria infection by collecting blood from finger prick. giemsa stained of thick and thin blood film smears were prepared to determine the presence or absence of malaria parasite.

\subsubsection{Measurement of Anemia}

The packed cell volume (PCV) was used as a simple screening test for anemia, as a reference method for calibrating automated blood count systems and as a rough guide to the accuracy of hemoglobin measurements. According to World Health Organization guidelines pregnant women are normal with hemoglobin concentration $11 \mathrm{~g} / \mathrm{dl}$ or $33 \%$ hematocrit value and above (10).

The micro hematocrit method is carried out on blood contained in capillary tubes $75 \mathrm{~mm}$ in length and having an internal diameter of about $1 \mathrm{~mm}$. The tubes may be plain for use with anti-coagulated blood samples. The centrifuge used for capillary tubes provides a centrifugal force of $12,000 \mathrm{~g}$, and 5minutes centrifugation result in a constant packed cell volume (PCV).

The micro hematocrit method has an adequate level of accuracy and precision for clinical utility (11).

\subsection{Data Analysis}

The data were entered, cleaned and edited using SPSS for windows version 16.0.Dependent variable frequencies, percentage, mean, range and proportion were calculated. The association between anemia (hematocrit $<33 \%$ or hematocrit $\geq 33 \%$ ) and its independent variable was examined by chi-square test analysis. Multivariate logistic regression analysis was used with variables significant at the $\mathrm{p}$ value $<$ 0.05 being kept in the final model. Variables which are biologically important and showed significant association in the previous studies included in multiple logistic regression analysis if in the chi-square test analysis; they had a $\mathrm{P}$ value of $<0.01 \%$.

\section{Results}

\subsection{Socio-demographic of Study Subjects}

Total of 748 informed and consented pregnant women who came for their antenatal follow up were enrolled in this study. The mean age of the attendants was 30 years old (range from 18-40). Seven (2.2\%) were 18 years old and five $(1.1 \%)$ were greater. The presence of anemia was assessed based on socio-demographic characteristics of the study subjects. Age, residence, occupation, family income, religion, marital status and educational status were taken as study variables to see the outcome of dependent variable. There was a statistical significant difference between all socio demographic variables and anemia except educational status with chi-square test analysis.

Access to drinking water was primarily from community installation, such as pipe water functioning only at certain times of the day. $250(66.8 \%)$ have a locally constructed latrine at a house hold level. The presence of anemia was assessed based on environmental related factors. Water and latrine were taken as a study variable to see the outcome of dependent variable. There was statistical significance difference between anemia and latrine.

Table 1. Distribution of intestinal parasites in pregnant women attending antenatal care at Sher-Ethiopia hospital center, 2014.

\begin{tabular}{|c|c|c|}
\hline Intestinal parasite & Number & Percent \\
\hline No ova or parasite & 312 & 41.7 \\
\hline Ascaris lumbricoides & 212 & 28.3 \\
\hline Trichuris trichuria & 20 & 2.7 \\
\hline Hook worm & 18 & 2.4 \\
\hline Schistosoma mansoni & 10 & 1.3 \\
\hline Hook worm with others & 110 & 14.7 \\
\hline Schistosomamansoni with others $*$ & 32 & 4.3 \\
\hline A.lumbricoides and T.trichuria & 32 & 4.3 \\
\hline A.lumbricoides with taenia spcies & 2 & 0.3 \\
\hline Total & 748 & 100 \\
\hline
\end{tabular}

Other* represents S.mansoni with A.lumbricoid and T.tricuria

\subsection{Parasitic Infection}

Intestinal parasites: parasitic infection among pregnant women was assessed and found that 436 out of 748 pregnant women were infected with intestinal parasites. Malaria was screened for all study subjects but no malaria cases were identified from all study subjects. Out of 436 helminthic infected women $80.4 \%$ ( 88 women) showed more than one intestinal helminthic infection. A. lumbricod was the leading 
parasite among pregnant women. The overall prevalence of $A$. lumbricoid in pregnant women was $54 \%$. The prevalence of anemia among intestinal helminthic infected and non-intestinal helminthic infected pregnant women was $70 \%$ and $29 \%$ respectively. (Table 2 )

The prevalence of anemia among pregnant women infected with hookworm plus other helminthes was $124 / 134(92.5 \%)$ whereas the prevalence of anemia among no intestinal helminthic infected women was 108/312(34.6\%), (table 2). This indicates that not being infected with intestinal parasite may reduce prevalence of anemia. The presence of anemia was assessed with intestinal parasite infection to see the outcome of the study variable. There was a statistical significant difference between Intestinal parasitic infection and anemia (Table- 2).

The overall prevalence of anemia in this study population using a cut off level $11 \mathrm{~g} / \mathrm{dl}$ (33\% hematocrit) was $51.9 \%$. The mean hematocrit level was 0.34 (34\%) range from $20 \%-41 \%$. Prevalence of anemia among rural women was higher than prevalence of anemia among urban women i.e., 318/580(54.8\%) and 70/168(41.6\%) respectively. The prevalence of anemia was $41.2 \%$, $56.5 \%$ and $59.5 \%$ for premigravida, multigravida and grand gravida respectively. The result showed that the occurrence of anemia increase with gravidity in that anemia is more prevalent in mothers with large number of pregnancies than mothers with few or one pregnancy. (Fig 1)

Table 2. Prevalence of anemia in relation to Helminthic infection in pregnant women attending antenatal care at Sher-Ethiopia hospital, 2014.

\begin{tabular}{|c|c|c|c|c|c|c|}
\hline \multicolumn{2}{|r|}{ Characterstics } & $\begin{array}{c}\text { Anemic } \mathrm{N}=194 \\
\text { No } \%\end{array}$ & $\begin{array}{c}\text { Non anemic } \\
\mathrm{N}=180\end{array}$ & Total number & Chi-square test value & $\mathrm{P}$-value \\
\hline \multirow{4}{*}{$\begin{array}{l}\text { Helminthic } \\
\text { infection }\end{array}$} & No ova or parasite & $54(27.8)$ & $102(56.7)$ & 156 & & \\
\hline & $\begin{array}{l}\text { Hook worm plus other } \\
\text { helminthes }\end{array}$ & $62(32)$ & $5(2.6)$ & 67 & 74.308 & $0.000 * *$ \\
\hline & $\begin{array}{c}\text { S.mansoni plus other } \\
\text { helminthes }\end{array}$ & $15(7.7)$ & $2(1.1)$ & 17 & & \\
\hline & All other helminthic infection & $64(33)$ & $70(38.9)$ & 134 & & \\
\hline
\end{tabular}

** Highly significant $(\mathrm{p}$ value $\leq .001)$

\section{Prevalence of anemia in pregnant women by their gravidity.}

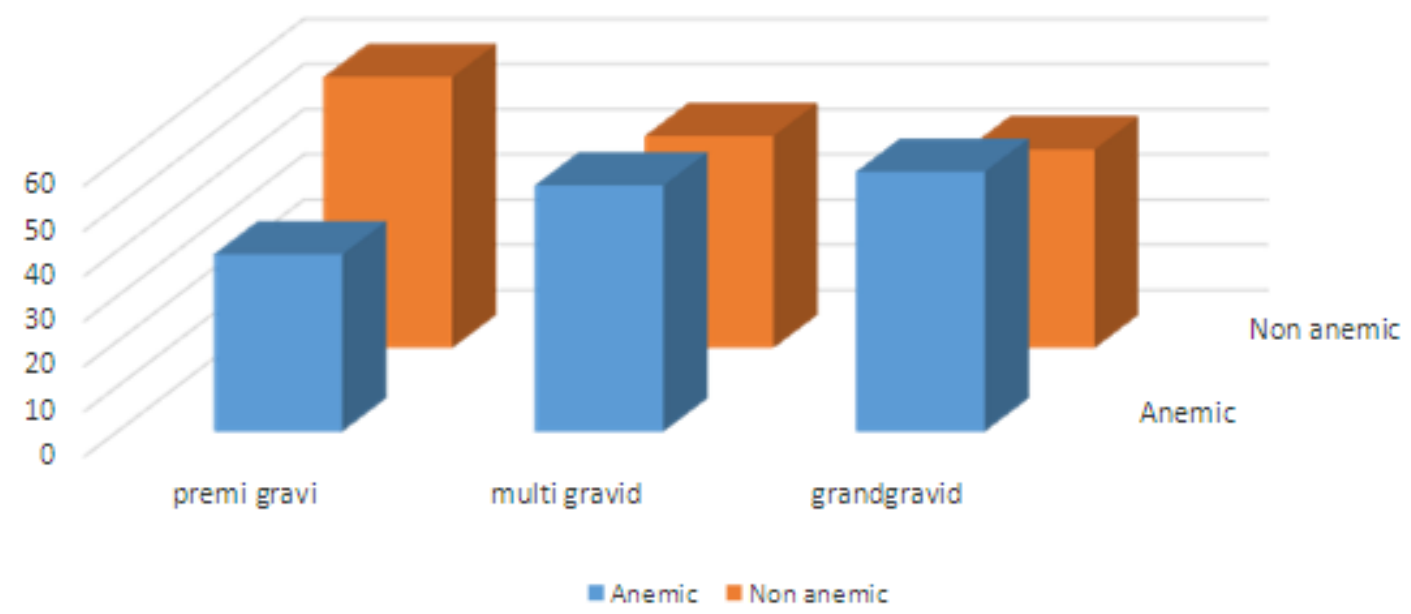

Figure 1. Prevalence of anemia in pregnant women by their gravidity during the study period at Sher-Ethiopia hospital, 2014. 


\section{Discussion}

Anemia is a common and serious problem in pregnancy. It needs to be addressed at community level as well as during antenatal care. Iron deficiency anemia has multifactorial causes in developing countries. The study has shown that anemia is prevalent in pregnant women attending antenatal follow up at Sher-Ethiopia hospital. The prevalence of anemia in the rural women was higher than prevalence of anemia in urban women. Similar results obtained in a study conducted at Asendabo and Mettue, anemia among pregnant women was consistently higher in rural women compare to the urban counter parts $(9,25)$. The prevalence of anemia obtained in this study $(51.9 \%)$ was almost consistent with the report of the previous studies $50 \%$ in Peru (12), $40-60 \%$ in developing countries pregnant women (13), but somehow lower prevalence of anemia compare to prevalence of anemia in pregnant women in Jimma(57\%) (14). anemia was also assessed at different kebles and high prevalence of anemia was found from Deset kebele antenatal care attendants. Deset kebele is located inside Ziway Lake and the area is endemic to $S$. msnsoni. The presence of $S$. mansoni in addition to other intestinal helminthes and less antenatal care service in the kebele may increase the prevalence of anemia at Deset kebele. There was low Prevalence of anemia in pregnant women who came from Kebele 02. Comparing to other kebeles there is a better antenatal care service and iron supplements at kebele 02 that may reduce the prevalence of anemia. On chi-square test analysis there was statistical significant differences between anemia and socio-demographic variables including residence, religion, occupation, marital status, monthly income, and latrine although only educational status showed a statistical significant difference with anemia on multivariate logistic regression analysis which indicates the lower chance of confounding effect of these variables on anemia. A study conducted on risk factors of anemia during pregnancy among pregnant women in India showed a statistical significant association between education and anemia which is consistent with this study (8). This finding indicates the need for strengthening of interventions related to education to women to create awareness of antenatal care, balanced diet during pregnancy and family planning. Parasitic infections contribute to iron deficiency in developing countries. The burden of disease imposed on helminthic-infected in child bearing age, especially during pregnancy may be one of single most important contribution of intestinal parasitic infections to the calculation of their global disease burden. The total amount of work a women can do in a day definitely decreases when she is anemic, whatever the cause is, and pregnancy plus helminthic infections produce double burden for women in some rural farming communities(15). Women may even acquire helminthic infection in the process of growing the family's food thus increase their degree of anemia in pregnancy.

The prevalence of intestinal parasites identified in this study $(58.2 \%)$ is higher than the prevalence reported for pregnant women in Congo (9\%), Nigeria (12.5\%), Mexico (38.2\%), Brazil (Sao Paulo State, 45.1\%) and lower than Indonesia (69.7\%) and Brazil (Rio dejaneiro State, 69\%) (16). This might be due to the difference in geographical location and economic status. The prevalence of individual intestinal parasite in pregnant women at Sher-Ethiopia hospital was recorded as, A.lumbricoid $28.3 \%$, hookworm $2.4 \%$, S.mansoni $1.3 \%$ (table 1 ).

A.lumbricoid was the leading parasite (48.8\%) followed by hookworm $(17.1 \%)$. The occurrence of helminthic infection at high rates among pregnant women is an indicative of faecal pollution of soil, improper utilization of latrine and poor personal hygiene in the study area. The prevalence of intestinal helminthic infection among pregnant women was $64.7 \%$. About $41 \%$ helminthic infected women had mixed infections, due to two or more than two different parasite species which might increase the burden and intensity of helminthic infection which could increase prevalence of anemia, almost twice as compare to those who did not infected with intestinal helminthes. In this study although all intestinal parasitic infections showed a statistical significance difference with anemia on chi-square test analysis, only hookworm plus other intestinal parasitic infection showed a statistical significant difference with anemia on multivariate regression analyses $(\mathrm{P}=.000)$, OR (95\% CI): $14.173(4.251,47.25)$. Those pregnant women infected with hookworm plus other intestinal parasitic infections were 14times likely to be anemic. Previous study report showed that an association between moderate and heavy hookworm infection and anemia was strengthened when there was co-infection with moderate and heavy T.trichuria infection (17 ).Another study also showed a significant association between hookworm infection and anemia $(18,20)$. Infections causing chronic blood loss such as parasitic infection with hookworm, increase iron requirement. Hookworm infection is described to be one of the principal causes of iron deficiency anemia. This finding indicates the need for strengthening of interventions related to screening intestinal parasitic infection for pregnant women during their first antenatal care service.

No malaria parasite was found in all study subjects. The reason may be the study period was not malaria season, indoor residual spraying of insecticides, the accessibility of bed net use for all kebele and no study subjects came with malaria clinical manifestation. On the assessment of anemia with pregnancy variables, there was a statistical significant difference between anemia and birth interval, gravida, taking iron tablets, shoe wearing, abortion and current regular antenatal care attendant on chi-square test analysis, but only birth interval, taking iron tablets, shoe wearing remain statistically significant difference with anemia on multivariate logistic regression analysis which indicates the lower chance of confounding effect of other variables on anemia. Pregnancy with a short birth interval leads to iron deficiency anemia as iron requirements are substantially higher than the average (19). Short birth interval increases risk for uterine rupture. There was a significant association 
between birth interval and anemia $(\mathrm{p}=.018)$, OR $(95 \% \mathrm{CI})$ : $2.180(1.141,4.166$.) This result was consistent with a study conducted in Jimma hospital (20).

Pregnant women with short birth interval less than or equal to two years were two times likely to be anemic. Appropriate time after each pregnancy for recuperation and replenishment of nutrient stores and circulating levels is a minimum of three to five years (27). This finding indicates the need for strengthening of interventions related to child spacing and awareness especially in rural women.

Iron deficiency occurs if the amount of iron absorbed is too little to meet the body's demand. This may be due to insufficient iron intake, reduce bioavailability of dietary iron intake and increase iron requirements during pregnancy. Malabsorption and loss of appetites due to intestinal parasitic infection may also cause iron deficiency. In this study there was a significance association between iron intake and anemia ( $p=.007)$, OR $(95 \% \mathrm{CI}): 2.60(1.29,5.23)$. This finding was consistent with the report of the previous study taking iron tablets significantly and positively associated with hemoglobin concentration in pregnant women (21). Pregnant women who did not take iron during pregnancy were greater than two times likely to be anemic than those who took iron supplement. WHO recommends that all pregnant women be supplemented with $60 \mathrm{mg}$ iron daily, in a pill that also usually contains $400 \mu \mathrm{g}$ folic acid (22). An additional benefit of iron supplementation during pregnancy is the opportunity to improve the maternal iron stores postpartum. This could reduce the risk of anemia during lactation and in subsequent pregnancy. There was a statistical significance difference between anemia and shoe wearing habit $(\mathrm{p}=.025)$, OR $(95 \% \mathrm{CI}): 2.19(1.10,4.33)$. Similar result was obtained in a previous study (20). Pregnant women walking barefoot were two times likely to be anemic. Most rural pregnant women attending antenatal care walk barefoot; even those women who have shoe do not wear regularly. They wear shoe when they come to town for antenatal care and for marketing. Walking barefoot may predispose to hookworm infection and the consequence will result iron deficiency anemia especially in pregnant women. Prevalence of anemia was high in the third trimester and grand gravida which indicates the need for proper follow up of pregnant women starting from the first trimester to the third trimester and the need of attention to grand gravida mothers. Prevalence of anemia among aborted mothers was much higher $(75 \%)$ as compare to non-anemic mothers which indicates that previous history of pregnant women should be taken during their first antenatal care visit to properly manage aborted mothers. Regular antenatal care attendant pregnant women showed low prevalence of anemia but, there was high prevalence of anemia $(74 \%)$ in non-regular antenatal care attendant pregnant women. Antenatal care initiated in the first trimester facilitates early diagnosis of anemia and allows treatment at the periphery so that the condition can be corrected before delivery. One of the major contributory factors in developing countries is consumption of plant based food containing insufficient iron, especially insufficient available haem iron from meat (23). Iron absorption is enhanced when consumed with foods high in vitamin $\mathrm{C}$ such as orange juice but substances in coffee and tea inhibit iron absorption (24). This study tried to assess different dietary risk factors for anemia. Eating animal food, eating green leafy vegetables and taking fruit after meal showed a statistical significance difference with anemia on chi-square test analysis but none of them showed a statistical significant difference with anemia on multivariate analysis which may be due to no difference in eating habit between anemic and non-anemic group.

\section{Conclusions}

From the result it can be concluded that high prevalence of anemia and intestinal helminthes among pregnant women in the study area. There was high prevalence of mixed intestinal parasitic infection among pregnant women. The prevalence of anemia was high in third trimester, grand gravida, aborted mothers and pregnant women who did not attend current antenatal care regularly. Anemia in pregnant women found to have association with: Hookworm plus other helminthes. Absence of taking iron during pregnancy and walking barefoot, birth interval less than or equal to two years, Less educational status.

\section{Ethics approval}

Every interviewee was informed about the objectives of the survey and their consent was obtained. Individual anemia status results were shown and explained to each participant. The study received ethical approval from Sher-Ethiopia hospital and the head office Ziway city administration.

\section{Acknowledgements}

Head office Ziway city administration is duly acknowledged for allowing me to do my study in Ziway town. I would like to thank Sher-Ethiopia hospital staffs who participated to conduct this research.

Last but not least thanks goes to all of my friends and family for their support in my academic life.

\section{REFERENCES}

[1] World Health Organization /UNICEF. (2001). Iron deficiency anaemia, assessment, prevention and control: a guide for programme managers

[2] World Health Organization. (1996). Report of the WHO informal consultation on hookworm infection and anaemia in girls and women. Geneva: WHO, CTD/SIP/96.1.

[3] Hercberg S., Galan P. (1992). Nutritional anaemia Baillieres Clin Haematol 5:143 - 168. 
[4] Sifakis S., Pharmackides G. (2000). Anaemia in pregnancy Ann NY Acad Sci, 90:125 - 136.

[5] Peter J., Simon B., Phil J., Bethony M., Maria E., Alex L., Shuva X. (2004). Hookworm infection, N Eng J Med, 351(8): $799-807$.

[6] Nurdia S., Sumarni S., Suyoko., Hakim M., Winkvist A. (2001). Impact of intestinal helminthes infection on anaemia and iron status during pregnancy: a community based study in Indonesia. SouthEast Asian J Trop Med Pub Health, 32 (1): $14-22$.

[7] Singh B., Fong F, Arikumeron S. (1998). Anaemia in pregnancy, a cross sectional study in Singapore, European $J$ Clin Nutr, 52(1) 65- 70.

[8] Bechuram M., Vikal T., Ranjan G. (2006). Risk factors of anaemia during pregnancy among the Garo of Meghalaya, India. Human Ecology Special Issue, 14(1):27-32

[9] [9] Tadios Y. (1996). Prevalence of anaemia and its risk factors among pregnant mothers following antenatal in Asendabo health center.

[10] World Health Organization. (1999). Monitoring Helminth Control Programs (WHO/CDS/CPC/SIP/99.3).

[11] World Health Organization. (2000). WHO recommended method for determination of packed cell volume by centrifugation prepared by Expert Panel on Cytometry of International Council for Standardization in haematology Document WHO, DIL 00219

[12] Zavaleta N., Berlanga R., Lonnerdal B., Brown H. (1993). Prevalence and determinants of iron deficiency anaemia in a representative sample of pregnant women in Lima, Peru,. Pan American Health Organization.

[13] World Health Organization. (1993). Prevention and management of severe anaemia in Pregnancy. WHO, $\mathrm{WHO} / \mathrm{FHE} / \mathrm{MSM} / 93-5$

[14] Desalegn S. (1993). Prevalence of anaemia in pregnancy in Jimma town, Southwestern Ethiopia. Ethiop Med J, 31:251-58.

[15] Stephenson S., Latham C., Ottesen A. (2000). Malnutrition and parasitic helminth infections. Parasitology 121: S23-S38.

[16] Alfonso J., Rodríguez M., Rosa A., Cynthia C., Melissa A., Marisela R., Henry P., Oscar U.,Gloria G., Nestor R, Andrea M.(2006). Intestinal Parasitic Infections amongPregnant Women in Venezuela. Infect Dis Obstet Gynecol. 10(1155):
23125.

[17] Mary A., Akanmori D. (2005). Determinants of anaemia in pregnancy in Sekyere West DistrictGhana. Ghana Med J, 39(3): 102-107

[18] Renee L., Martin C., Edduardo G., Theresa G. (2005). Relation ship between intensity of soil - transmitted helminthes infection and anaemia. During pregnancy. $A M J$ Trop Med Hyg, 73(4): 783 - 789.

[19] World Health Organization. (1991). Controlling iron deficiency. ACC/SCN state of the art series Nutrition Policy Discussion Paper No.9.

[20] Belachew T., Yosef L. (2006). Risk factors for anaemia among pregnant women attending antenatal clinic at Jimma University Hospital, Ethiop Med J, 44, No.3.

[21] Aikawa R., Ngyen K., Sasaki S., Binns W. (2006). Risk factor for iron deficiency anaemia among pregnant women living in rural Vietnam. Pub Health Nutr. 9(4): 443-8.

[22] Stoltzfus J., Drefuss L. (1998). Guidelines for the use of iron supplement to prevent and treat iron deficiency anaemia. Geneva: International nutrition anaemia consultative group /UNICEF/ WHO

[23] Van den Brock R. (2003). Anaemia and micronutrient deficiencies. British Med Bull, 67: 149- 160.

[24] Cook J., Monsen R. (1997). Vitamin C, the common colds and iron absorption. Am J Clin Nutr, 30:235-241.

[25] Gebremedin S. (2004). Prevalence of anaemia and its predisposing factors in pregnant mothers attending antenatal care in Mettu Carl hospital.

[26] Monica C. (1998). District Laboratory Practice in Tropical Countries, Part one. PP 99-100.

[27] Reproductive Health. New findings on birth spacing: three to five years is the optimal interval,http://www.infoforhea lth.org/pr/113edsum.shtmle server programs birth spacing optimal interval. Date of accessed October 5, 2008

[28] Wodimanehu T., Birrie H., Yemaneh H. (1997). Schistosomiasis and intestinal helminthic infections in Delo Awraja, Bale administrative region South Ethiopia. Ethiop $J$ Health Dev. 11(3): 183 -188.

[29] Bethony J. (2002). Emerging patterns of hookworm infection: influence of aging on the intensity of Necator infection in Hainan Province, People's Republic of China. Clin. Infect. Dis. 35:1366-1344. 